\title{
Optimizing transformations of structural balance in signed networks with potential relationships
}

\author{
Haifeng Du ${ }^{1}$ Xiaochen $\mathrm{He}^{1}$ Shanfeng Wang ${ }^{2}$ Maoguo Gong ${ }^{2}$ Marcus W. Feldman ${ }^{1,3 *}$ \\ ${ }^{1}$ Center for Administration and Complexity Science of Xi'an Jiaotong University, Xi'an, Shanxi \\ Province, 710049 \\ ${ }^{2}$ Key Laboratory of Intelligent Perception and Image Understanding of Ministry of Education, \\ Xidian University, Xi'an, Shanxi Province 710071, \\ ${ }^{3}$ Morrison Institute for Population and Resource Studies, Stanford University, Stanford, CA 94305
}

*Corresponding author

The research was supported by the Key Project from the National Science Foundation of China (Grant No. 12AZD110), the Fundamental Research Funds for the Central Universities (Grant No.2011jdgz08) and the National Natural Science Foundation of China (Grant No. 71071128)

June 17, 2016 
Abstract: A signed network includes positive edges, negative edges and " 0 " edges, the last of which denote potential relationships. However, " 0 ” edges are commonly ignored in transformations of unbalanced networks. In this paper, we take "0" edges into account and solve the optimization problem in a more comprehensive way. We transform the unbalanced network by not only changing signs of edges but also changing edges into potential. The experimental results show that our method can solve this problem efficiently, and that our solutions are cost-saving.

Keywords: structural balance, signed networks, transformation, optimization 


\section{Introduction}

A signed network can be defined as a graph $G=(V, R, \sigma)$ where $V$ is the set of nodes, $R$ is the set of edges and $\sigma$ is a sign function. The nodes $\left\{v_{i}\right\} \in V$ represent the individuals while the edges between pairs of nodes $\left(v_{i}, v_{j}\right) \in R$ represent the relationships between those individuals. $\sigma: R \rightarrow\{+1,-1\}$ gives the signs of those edges, where +1 denotes positive relationships and -1 denotes negative relationships [1].

A complete signed network has exact structural balance if and only if every triangle has three +1 (positive) edges or only one +1 edge [2]. Structural balance is an important property of a signed network. Heider first developed the theory of structural balance in social psychology and used it to detect the origin of conflicts in a signed network [2]. Heider's theory was modified by Cartwright and Harary, who used graph theory to analyze the structural balance of a signed network. However, their theory related only to complete networks [3]. Easley and Kleinberg proposed a more general definition of structural balance for arbitrary networks [4]. Arbitrary networks are more realistic because they include not only positive or negative relationships but also potential relationships whose signs are not known, which means that the sign function for edges, $\sigma: R \rightarrow\{+1,-1\}$, should become $\sigma: R \rightarrow\{+1,0,-1\}$. The ' 0 ' edges denote potential relationships; potential relationships are generally more common than signed relationships because actual networks are usually sparse. According to the theory of Easley and Kleinberg, an arbitrary network can be "completed" by changing the 0 
edges to -1 or +1 .

In the real world, most networks are not balanced. Therefore, transforming unbalanced networks in order to achieve global balance is important [5]. Antal et al. proposed a discrete-time model in which unbalanced triangles evolved into balanced ones by changing signs of edges [6]. However, the model always falls into unbalanced states, which they called "jammed states". To solve this problem, Kulakowski et al. [7] proposed the continuous-time model shown in Eq. 1 to explain the process of changing an initial adjacency matrix into a balanced adjacency matrix. In Eq. $1, x_{i j}$ denotes the edge between nodes $i$ and $j$, while $t$ denotes time.

$$
\frac{d x_{i j}}{d t}=\sum_{k}^{n} x_{i k} x_{k j}
$$

The continuous-time model has since been applied and improved by many scholars [8-10]. In [8], the initial matrix reached a balanced state with probability converging to 1 in finite time, and a closed-form expression for the balanced state, in contrast to the initial state, was provided by Marvel et al. [8]. In [9], a model was proposed to explore asymmetric networks. Summers and Shames studied active external influence and showed that perturbing the local friendliness level of a random initial network can achieve the desired structural balance [10].

Transformation of unbalanced networks can also be regarded as an optimization problem. Facchetti et al. introduced an energy function to compute the structural balance [11]. The minimum value of the energy function represents the lowest number of edges that leads to global imbalance, and when the energy function equals 0 , the network is balanced. Some heuristic algorithms, such as the genetic algorithm, 
simulated annealing, and tabu-search [12-14], have been applied to solve this NP-hard problem [15].

These dynamical models and optimization algorithms all proceeded by changing signs of edges to achieve a balanced network. However, the social significance of these operations has not really been considered. On one hand, 0 edges (potential relationships) are ignored during the dynamical or optimization process. On the other hand, the transformation cost of changing an edge from +1 to -1 is different from a change from -1 to +1 in the real world. If 0 edges are included, the situation will become more complex. Obviously, there are six different situations for edge changing, +1 to $-1,-1$ to $+1,+1$ to 0,0 to $+1,-1$ to 0,0 to -1 . The +1 relationship (such as friend) changed to 0 (uncertain or potential) is different from changing -1 to 0 .

In this paper, we solve the problem by changing edges from +1 to -1 , from -1 to +1 , from +1 to 0 , and from -1 to 0 . Changes from 0 to +1 and 0 to -1 are not included because 0 represents potential relationships that cannot be observed in the initial network. Further, changes from 0 to +1 or -1 entail adding edges, in which case 0 would represent nonexistent relationships rather than the potential relationships. We introduce a new energy function to measure the unbalanced level of a signed network, and a memetic algorithm is employed to optimize the transformation of unbalanced networks. The rest of this paper is organized as follows. Related research concerning structural balance is discussed in section 2; section 3 explains the optimization algorithm; applications and experiments are shown in section 4. Our conclusions are presented in section 5 . 


\section{Related Background}

\subsection{Structural balance}

Following Heider [2], Fig. 1 shows all four possible triangles. The triangles in Fig. 1(a) and (b) are balanced and consist of an even number of -1 (negative) edges, while the triangles in Fig. 1(c) and (d) are unbalanced and consist of an odd number of -1 edges. The two unbalanced triangles require some latent force to change some edges.

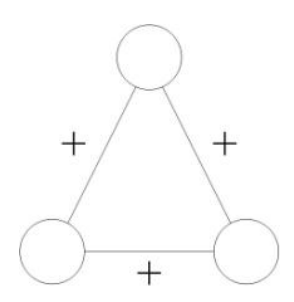

(a)

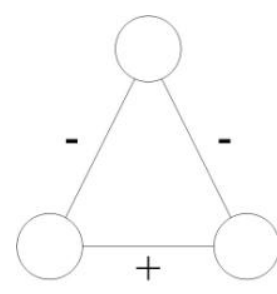

(b)

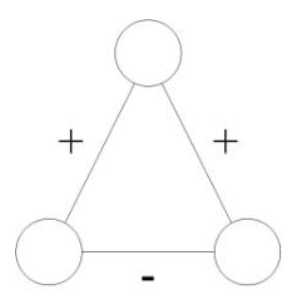

(c)

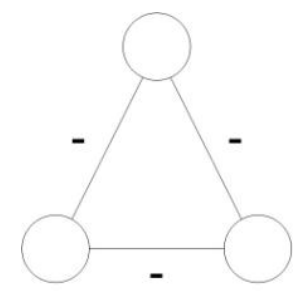

(d)

Fig. 1. All possible triangles in a network. Edges with ' + ' and '-' signs represent friendly and unfriendly relations, respectively. The triangles in (a) and (b), which have an even number of negative edges are balanced; they comply with the logic "my friend's friend is also my friend" and "my friend's enemy is also my enemy". The triangles in (c) and (d), which have odd numbers of negative edges, are unbalanced.

Based on graph theory, Harary divided all the nodes of a complete signed network into two clusters; if every pair of nodes belonging to the same cluster are connected by +1 edges and every pair of nodes belonging to different clusters are connected by -1 edges, then it is a balanced network [3]. In fact, Harary's definition of structural balance is equivalent to Heider's, and they are both definitions of strong balance [4]. A weaker form of balanced network can be divided into more than two clusters, but in this paper we analyze only the strong form of balance.

To generalize the idea of structural balance in an arbitrary network $G=(V, R, \sigma), \sigma: R \rightarrow\{+1,0,-1\}$, Easley and Kleinberg propose two equivalent ways 
of defining structural balance [4]. First, an arbitrary network is balanced if it can be "completed" by filling in the missing edges (denoted by 0 ) to produce a balanced complete network. Second, they view structural balance as dividing an arbitrary network into two mutually opposed sets of friends. In this paper, we adopt the second definition of balance to apply our algorithm, but we note that missing edges are also important for transforming an unbalanced network into a balanced one. Actually, a missing edge represents the potential relationship between two nodes and can be changed to +1 or -1 as needed.

\subsection{Transformations of unbalanced networks}

There are two ways to transform an arbitrary unbalanced network $G=(V, R, \sigma), \sigma: R \rightarrow\{+1,0,-1\}$ into a balanced one. The popular way is to change a relationship that leads to global imbalance into its opposite, namely, to change the enemy into a friend or a friend into an enemy. The other way, borrowed from Easley and Kleinberg's "filling-in" edges, is to change the real relationship (+1 or -1 edge) into a potential one (0 edge), which is equivalent to "filling in" an edge. Then the relationship between two individuals enters a special state; they are neither friends nor enemies; that is, the relationship is removed.

The combination of these two procedures contains more possible solutions. Fig. 2 shows an unbalanced network in which the solid lines with ' + ' denote +1 edges and the solid lines with '-' denote -1 edges. If we change the unbalanced edges into their opposites, we have three best choices to make the network balanced: we could turn A into -1 and $\mathrm{B}$ into +1 ; we could turn $\mathrm{B}$ and $\mathrm{C}$ into +1 ; we could turn $\mathrm{D}$ and $\mathrm{E}$ into -1 . 
But if we are permitted not only to turn the unbalanced edges into their opposites but also to change an edge into potential, we could have 12 best solutions including the three solutions mentioned above. If we assign a cost to each of the two solutions, their total cost may be different; it is possible that with alternative solutions, more lower-cost solutions could be available.

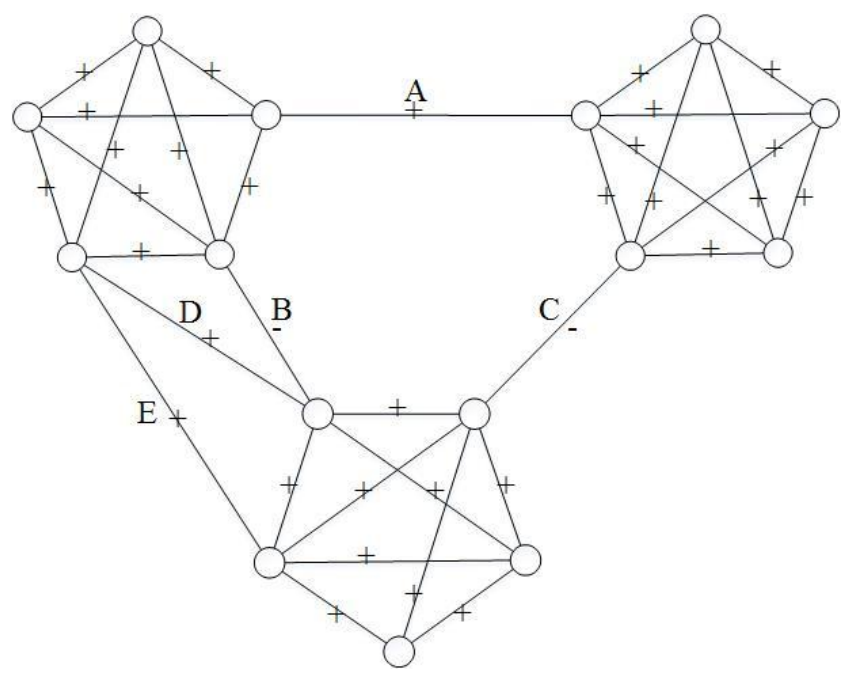

Fig. 2. An illustration of the combination of the two changing procedures. Lines with '+' denote positive relations and lines with '-' denote negative relations.

\subsection{The energy function}

Facchetti proposed an energy function $H(s)$ to measure the imbalance level of a network $G=(V, R, \sigma), \sigma: R \rightarrow\{+1,-1\}[11]$ :

$$
H(s)=\sum_{(i, j)}\left(1-a_{i j} s_{i} s_{j}\right) / 2
$$

where $i, j=1,2, \ldots n, i \neq j$ represent two different nodes in a network; $a_{i j} \in\{-1,1\}$ denotes the relationship between node $i$ and node $j$, where $a_{i j}=-1$ is enemy, and $a_{i j}=1$ is a friendly relationship; $s_{i} \in\{-1,1\}$ represents the cluster that node $i$ belongs to, where $s_{i}=1$ or $s_{i}=-1$ represent the two different clusters.

The minimum of $H(s)$ gives the least number of edges whose signs should be 
changed in order to achieve the global balance. In particular, $H(s)=0$ indicates the network is balanced. If we include 'missing' edges in a network, namely, $G=(V, R, \sigma), \sigma: R \rightarrow\{+1,0,-1\}$, the energy function $H(s)$ should be modified to become

$$
E(s)=\sum_{(i, j)}\left(\left|a_{i j}\right|-a_{i j} s_{i} s_{j}\right) / 2
$$

where now $a_{i j}$ in Eq. 2 becomes $a_{i j} \in\{-1,0,1\}$, and $a_{i j}=0$ denotes that the relationship is neither friend nor enemy.

If nodes $i$ and $j$ belong to the same cluster $\left(s_{i}=s_{j}\right)$ and they are connected by a positive edge $\left(a_{i j}=1\right), E(s)$ doesn't increase; if they are connected by a negative edge $\left(a_{i j}=-1\right), E(s)$ increases by 1 , which represents an increase in level of imbalance; if they have no connection or they are neither friends nor enemies $\left(a_{i j}=0\right), E(s)$ remains unchanged. When nodes $i$ and $j$ belong to different clusters $\left(s_{i} \neq s_{j}\right)$, and they are connected by the positive edge $\left(a_{i j}=1\right), E(s)$ increases by 1 ; if they are connected by a negative edge $\left(a_{i j}=-1\right)$ or by a 0 edge $\left(a_{i j}=0\right), E(s)$ doesn't increase. The minimum of $E(s)$ is the lowest number of edges that should be changed from +1 to $-1,-1$ to $+1,+1$ to 0 , or -1 to 0 in order to achieve global balance.

\subsection{Quantifying transformations of unbalanced networks}

The problem of optimizing transformations to achieve structural balance can be mathematically formulated as

$$
\min F(x)={ }_{1} X_{+\rightarrow}+{ }_{2} X_{\rightarrow+}+{ }_{3} X_{+\rightarrow 0}+\left(\begin{array}{llll}
1 & 1 & 2 & 3
\end{array}\right) X_{\rightarrow 0}
$$

such that $E(s)=0$. In order to reduce the cost, we take minimizing $F(x)$ as our objective. Here $X_{+\rightarrow-}$ represents the number of positive edges that are changed into 
negative ones, $X_{-\rightarrow+}$ represents the number of negative edges that are changed into positive ones, $X_{+\rightarrow 0}$ represents the number of positive edges that are reassigned to 0 , $X_{-\rightarrow 0}$ represents the number of negative edges which have been reassigned to 0 , while $\omega_{1}, \omega_{2}, \omega_{3}$ and $\left(1-\omega_{1}-\omega_{2}-\omega_{3}\right)$ are corresponding weights assigned to the four variables mentioned above.

Evolutionary algorithms have been used previously to solve NP-hard computational problems. Gong et al. combined a genetic algorithm with an interactive algorithm to study networks in which the parameters identifying and connecting nodes had uncertainties. They applied their method to optimizing an interior layout [16]. A different approach was employed by Xuan et al. [17], who focused on modeling customer profits and the cost of requirements for the next release of a software development, the next release problem (NRP). This computational problem is also NP-hard even in its simplest form, and Xuan et al. [17] proposed a backbone-based multilevel algorithm (BMA), which employed multilevel reductions to downgrade the problem scale and multilevel refinements to construct the final optimal set of customers. This method allowed the problem to be partitioned into a set of smaller tractable problems.

The optimization of transformations of unbalanced networks is an NP-hard problem and is difficult to solve by traditional algorithms. Here, we use a memetic algorithm (MA), which, combined with a genetic algorithm (GA) and a local search strategy, has proved to be efficient in solving many computational problems $[18,19]$. 


\section{The Algorithm}

3.1. The framework of the proposed algorithm

The framework of our algorithm, shown in Algorithm 1 below, is similar to the framework in [20], but the InitialPopulation(), GeneticOperation() and LocalSearch() are quite different. We will describe these in detail. For other functions, Selection() is used to select the parent population for genetic operations; UpdatePopulation() is used to reconstruct a new population, consisting of better chromosomes, from the initial population $P$ and the offspring population $P_{\text {child }}$ after Selection(), GeneticOperation() and LocalSearch(). Termination() is the criterion to terminate the algorithm.

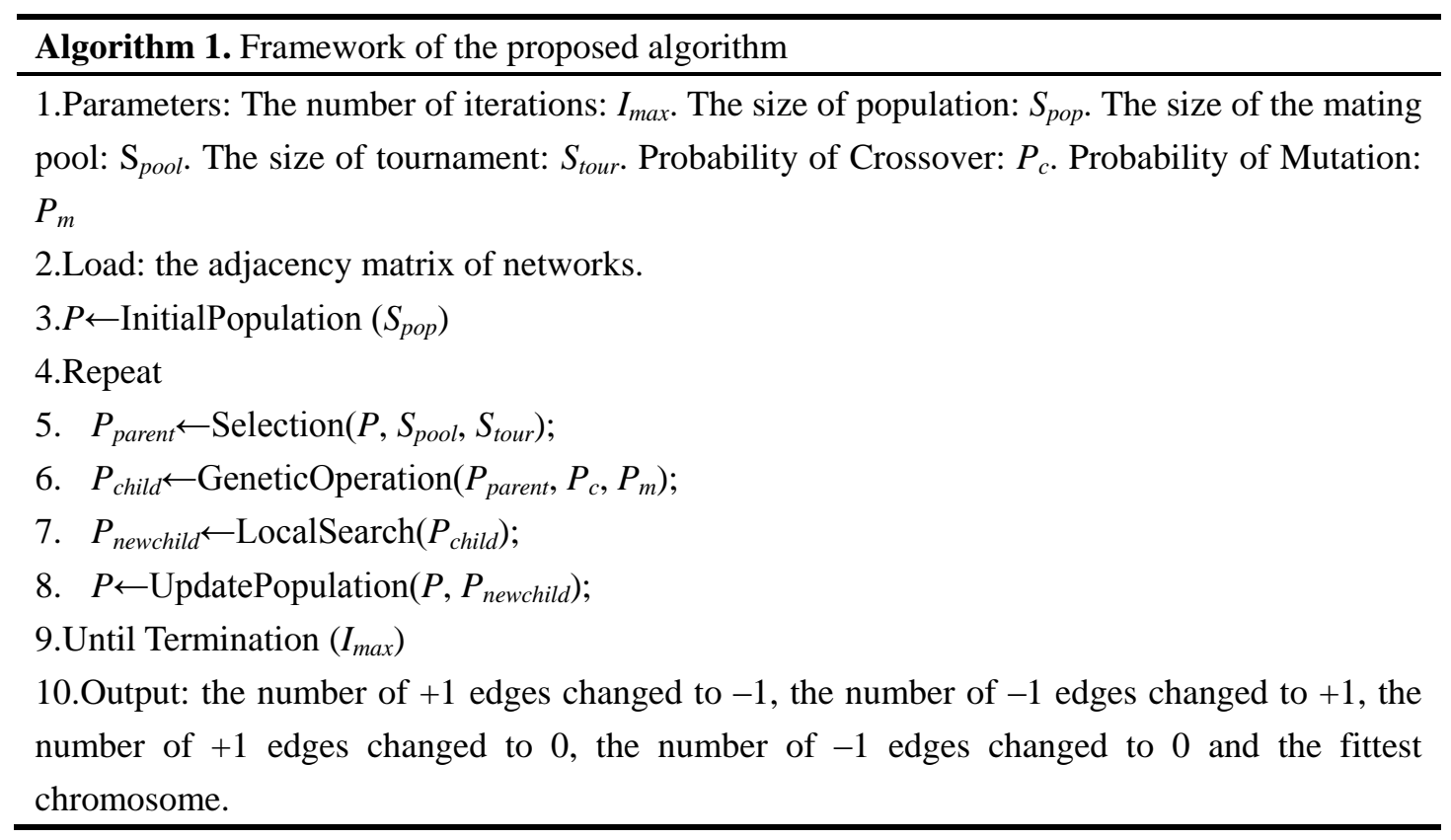

From Eq. 4, we take $\omega_{1}=\omega_{2}=\omega$ and $\omega_{3}=\left(1-\omega_{1}-\omega_{2}-\omega_{3}\right)=0.5-\omega$. The objective function is then:

$$
\min F(x)=\omega X_{+\rightarrow-}+\omega X_{-\rightarrow+}+(0.5-\omega) X_{+\rightarrow 0}+(0.5-\omega) X_{-\rightarrow 0}+\mu E(s)
$$

Here, $\mu$ is a penalty parameter, and with Eq. 5 the constrained optimization problem described by Eq. 4 is converted to an unconstrained one. 


\subsection{Representation and initialization}

Every individual can be encoded as a chromosome and each chromosome in the population consists of three substrings: $X=\left\{X_{p o s}, X_{n e g}, X_{\text {node }}\right\}$, where $X_{\text {pos }}$ indicates the vector of +1 edges in the initial network; $X_{\text {neg }}$ indicates the vector of -1 edges in the initial network; $X_{\text {node }}$ is the vector of nodes $x_{i}$ with $x_{i}=+1$ or -1 . If $x_{i} \subseteq\left\{X_{p o s}, X_{n e g}\right\}, x_{i}=1$ means the corresponding edge has not been changed, $x_{i}=0$ means the corresponding edge has been changed to potential, while $x_{i}=-1$ means the sign of corresponding edge has been changed. In Fig. 3, the initial chromosome consists of 12 genes. The first three genes represent the vector of +1 edges of the initial network, the fourth to seventh genes represent the vector of -1 edges, while the last five genes represent the vector of nodes. To change the -1 edge between node 1 and node 5 , we change the $6^{\text {th }}$ gene of the chromosome; Figs. 3(a) and Fig. 3(c), respectively, show this transformation of changing signs of edges and changing edges into potential.

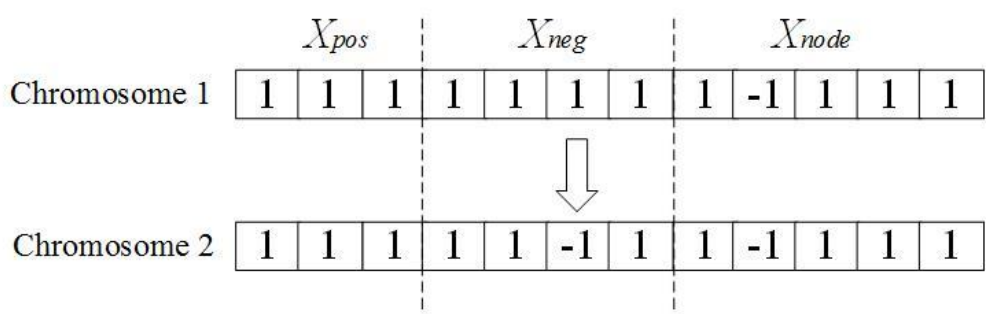

(a) A chromosome transformation changing a relationship to its opposite.
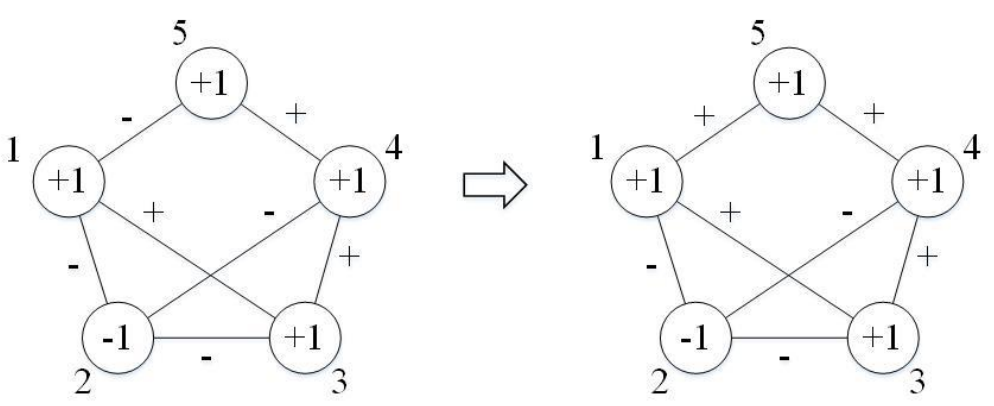

(b) Effect of changing a relationship to its opposite. 


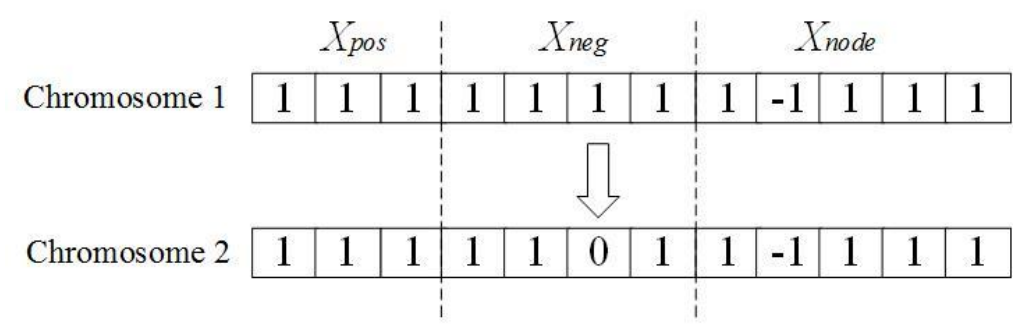

(c) Chromosome transformation changing a relationship to potential.
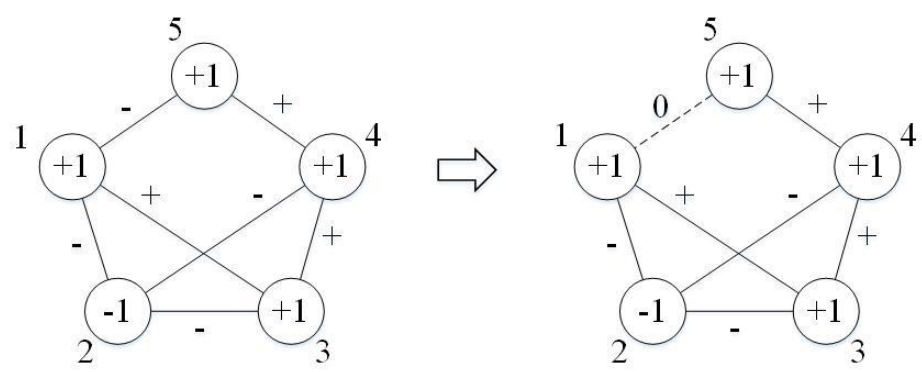

(d) Effect of changing a relationship into potential.

Fig. 3. Figs. 3(b) and 3(d) represent the effects of changing Figs. 3(a) and 3(c), respectively. In Figs. $3(\mathrm{a})$ and $3(\mathrm{c})$, the vectors are chromosomes consisting of three substrings: $X_{\text {pos }}, X_{\text {neg }}, X_{\text {node }}$. In Figs. 3 (b) and 3(d), the number near the node is the sequence number of the node. The solid lines with ' + ' denote positive edges, the solid lines with '-' denote negative edges, the dotted lines with ' 0 ' denote potential edges. " +1 " and " -1 " in the node indicate the node belongs to different cluster. The nodes ' 1 ', ' 3 ', ' 4 ', ' 5 ' are in the same cluster, and node ' 2 ' is in the other cluster. In Fig. 3(a), the sixth gene was changed from 1 to -1 , so the corresponding edge in Fig. 3(b) was changed from negative to positive. In Fig. 3(c), the sixth gene was changed from 1 to 0 , so the corresponding edge in Fig. 3(d) was changed from negative to potential.

Before we describe the population initialization algorithm in detail, we first give a definition of neighbors: If node $i$ is connected to node $j$ by a +1 edge, then we say node $j$ is a neighbor of node $i$.

Each chromosome in the population consists of three substrings: $X=\left\{X_{p o s}, X_{n e g}, X_{\text {node }}\right\}$, where the first two substrings denote the vector of edges, and the last substring denotes the vector of nodes. When we produce the chromosome, the first two substrings consist of genes $x_{i} \in\{-1,0,1\}$ while the last substring consists of genes $x_{i} \in\{-1,1\}$. In order to speed up the convergence, we introduce a greedy strategy to produce a higher-quality population. We randomly select a neighbor of one node and 
change the neighbor's value to the latter. The initialization procedure is showed in Algorithm 2.

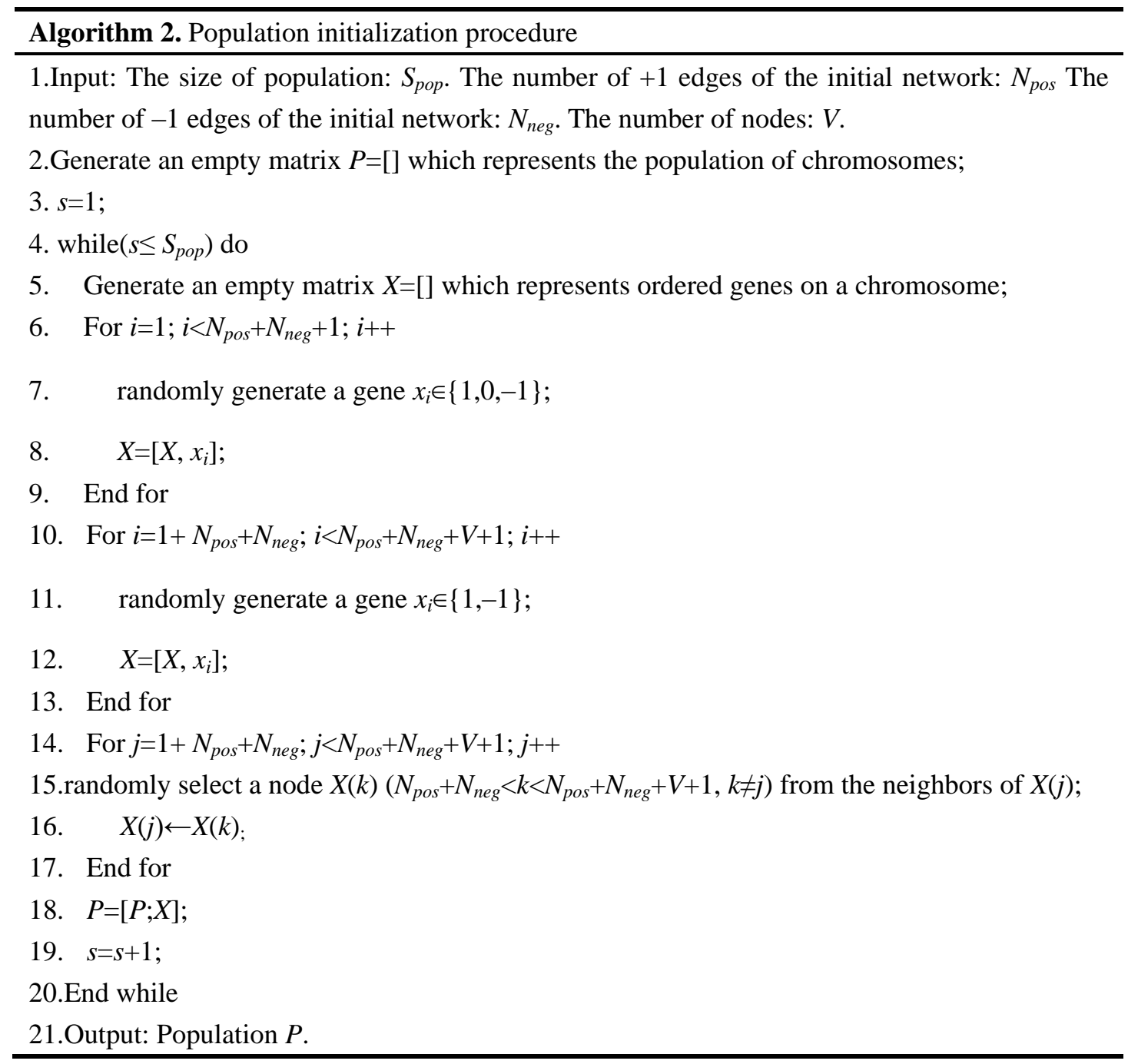

3.3. Genetic operation and local search

The genetic operation consists of two parts. The first is crossover. We randomly select two parent chromosomes $X^{a}$ and $X^{b}$, and randomly select two points in each substring $X_{\text {pos }}, X_{\text {neg }}$ and $X_{\text {node }}$ of $X^{a}$ and $X^{b}$. Then, the genes between these interior points of $X^{a}$ and $X^{b}$ are exchanged, and two new chromosomes produced. Fig. 4 shows the process of crossover. The second is mutation. We first randomly select a parent chromosome for mutation and assume one point mutation on it. For a gene 
$x_{i} \subseteq\left\{X_{p o s}, X_{n e g}\right\}$, we change its sign. For a gene $x_{i} \subseteq\left\{X_{\text {node }}\right\}$, we change its value to that of one of its neighbors chosen at random.

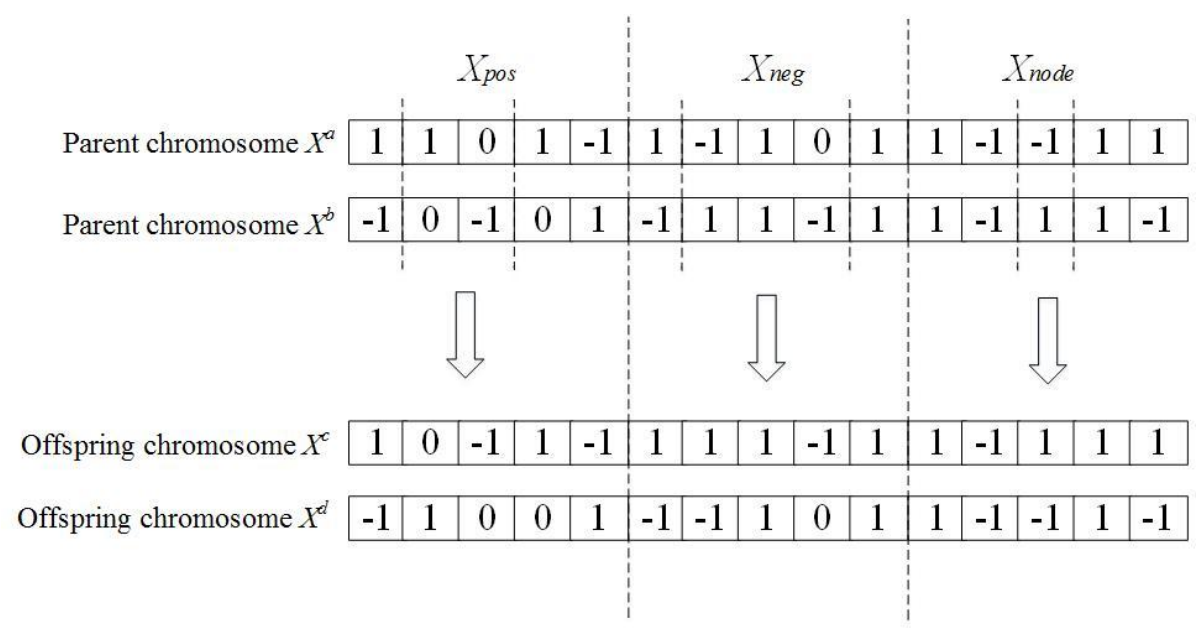

Fig. 4. The process of two-point crossover. All the chromosomes consist of three substrings: $X_{p o s}$, $X_{n e g}, X_{\text {node }}$. The two offspring chromosomes are the results of crossover between the two parent chromosomes by exchanging the genes between the vertical lines within each segment.

For the local search, we apply a local search strategy to the chromosome $X_{\text {child }}$ after the genetic operation. We select every gene $x_{i}$ from the chromosome and change it, and then check whether the change decreases the energy function. Then we choose the gene which gives the lower value of $E$. If the gene $x_{i}$ is located in the first two substrings, and if $x_{i}=1$ or $x_{i}=-1$, we have two changing strategies: we change the sign of the gene or we assign 0 to it. Since both these changes may give better results, we introduce a parameter $p_{1}$ of choosing the former. If $p_{1} \geq 2 \omega$ and the value of the energy function can decrease by changing the sign of the gene, we accept the new gene by changing its sign; if $p_{1}<2 \omega$ and the value of energy function can decrease by reassigning 0 to the gene, we accept the new gene changed by assigning 0 to it. If $x_{i}=0$, we also have two changing strategies: assign +1 to the gene or assign -1 to it. Here, we introduce a parameter $p_{2}$ : if $p_{2} \geq 2 \omega$ and assigning 1 to $x_{i}$ does not increase the value of energy function, we accept 
the new gene changed by assigning +1 to it, while if $p_{2} \geq 2 \omega$ and assigning -1 to $x_{i}$ does not increase the value of $E$, we accept the new gene changed by assigning -1 to it. For the gene $x_{i}$ located in the last substring, we have just one changing strategy, namely change the sign of the gene. If this decreases $E$, we accept the new gene. The local search procedure is showed in Algorithm 3.

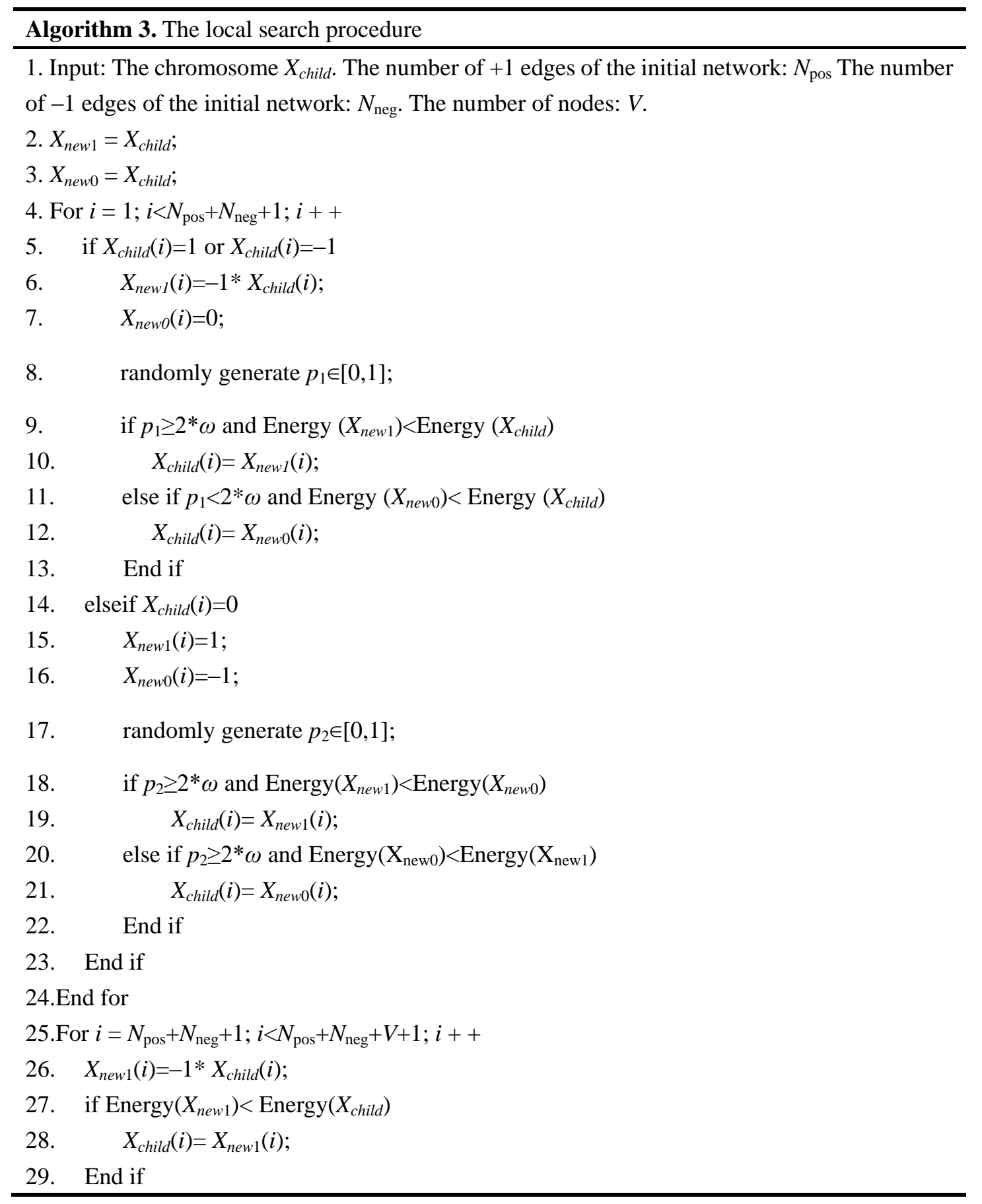


30.End for

31.Output: $X_{\text {child }}$;

\section{Experiments}

In this section, we show results of testing our algorithm on one computer-generated and four real-world networks. These results show that optimization of transformations of unbalanced networks can be achieved by our algorithm.

We used MATLAB to write our algorithm, and carry out our experiments on a 2.53 GHz CPU and 2.00 GB memory computer, operating on Windows 7. Table 1, lists the parameters used in this paper.

Table 1. The parameter of our algorithm.

\begin{tabular}{lll}
\hline Parameter & Meaning & Value \\
\hline$I_{\max }$ & Iterations & 300 \\
$S_{\text {pop }}$ & Size of population & 200 \\
$S_{\text {pool }}$ & Mating pool size & 100 \\
$S_{\text {tour }}$ & Size of tournament & 2 \\
$P_{c}$ & Probability of Crossover & 0.9 \\
$P_{m}$ & Probability of Mutation & 0.1 \\
\hline
\end{tabular}

4.1. Experimental results of the generated network

In order to explain the difference between changing signs of edges and changing edges into potential, we first generated an arbitrary unbalanced network (GN) consisting of 15 nodes, 33 positive edges and two negative edges as shown in Fig. 5a. In these experiments, $\mu$ and $\omega$ are not fixed values, and we want to know the effect of changing $\omega$. Thus we choose $\mu=1$, which denotes the cost of constraint, and $\omega=0.05$, $0.1,0.15,0.2,0.25,0.3,0.35,0.4,0.45$, which relates to the cost of different changing 
operations. Fig. 5b shows two solutions of changing edges into their opposites. The solid lines with '+' represent positive edges, and solid lines with '-' represent negative edges. The left graph in Fig. 5b shows that two positive edges are changed into negative ones and then the whole network is divided into two clusters; the right graph shows that two negative edges are changed into positive and all edges in the network become positive. Fig. $5 \mathrm{c}$ shows one of the solutions of changing edges into potential, where the dotted lines with ' 0 ' represent the potential edges; we see the two positive edges which lead to global imbalance are changed into potential. Another solution of changing edges into potential is similar to the right graph in Fig. 5b. There may be solutions provided by hybrid procedures, but we do not enumerate all the solutions. Although the corresponding edges changed by the two procedures are the same, the cost of these two sets of changes cannot be ignored.

Table 2 shows results produced using the operation with potential relationships (i.e. including 0 edges) and the operation without potential relationships. We ran our algorithm 20 times on the GN network; $N_{p n}, N_{n p}, N_{p 0}, N_{n 0}$, and $F$, respectively, denote the mean number of +1 edges that changed to -1 , the mean number of -1 edges that changed to +1 , the mean number of +1 edges that changed to 0 , the mean number of -1 edges that changed to 0 , and the mean value of the objective function. We can see that the objective function of transformations including 0 edges is smaller (not all $\omega$ values) than the objective function without 0 edges, especially for larger values of $\omega$. In this case, transformations of unbalanced networks including 0 edges will produce lower-cost solutions than those that do not including 0 edges. Further, we see that $N_{p n}$ 
and $N_{n p}$ decrease as $\omega$ increases, while $N_{p 0}$ and $N_{n 0}$ increase as $\omega$ increases if we include the potential relationships. This is because $\omega$ is directly related to the cost of changing signs of edges and changing edges into potential, and if the cost of either is large, the algorithm will choose that procedure with the smaller cost. In this way, the algorithm will choose the lowest-cost solution in attempting to achieve the global balance.

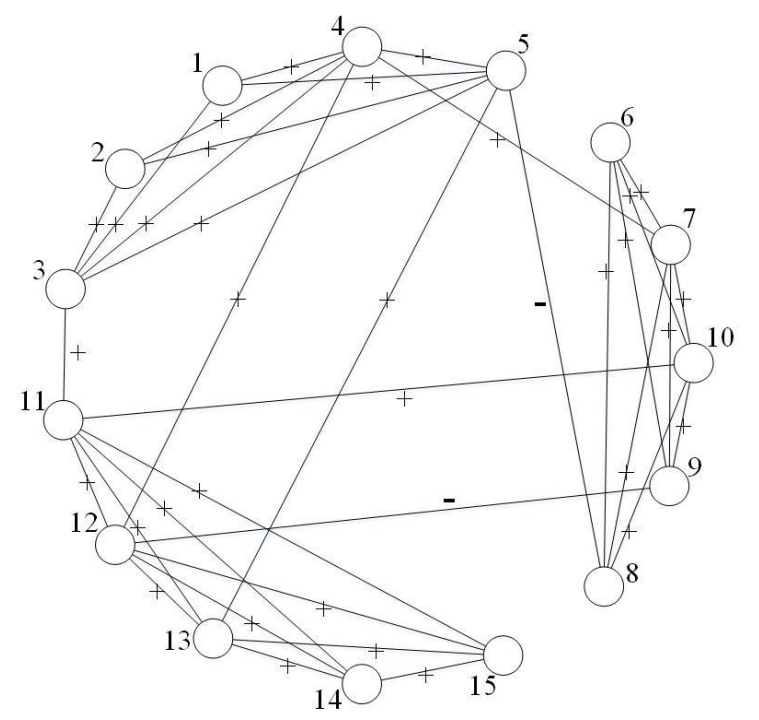

(a) The original GN network
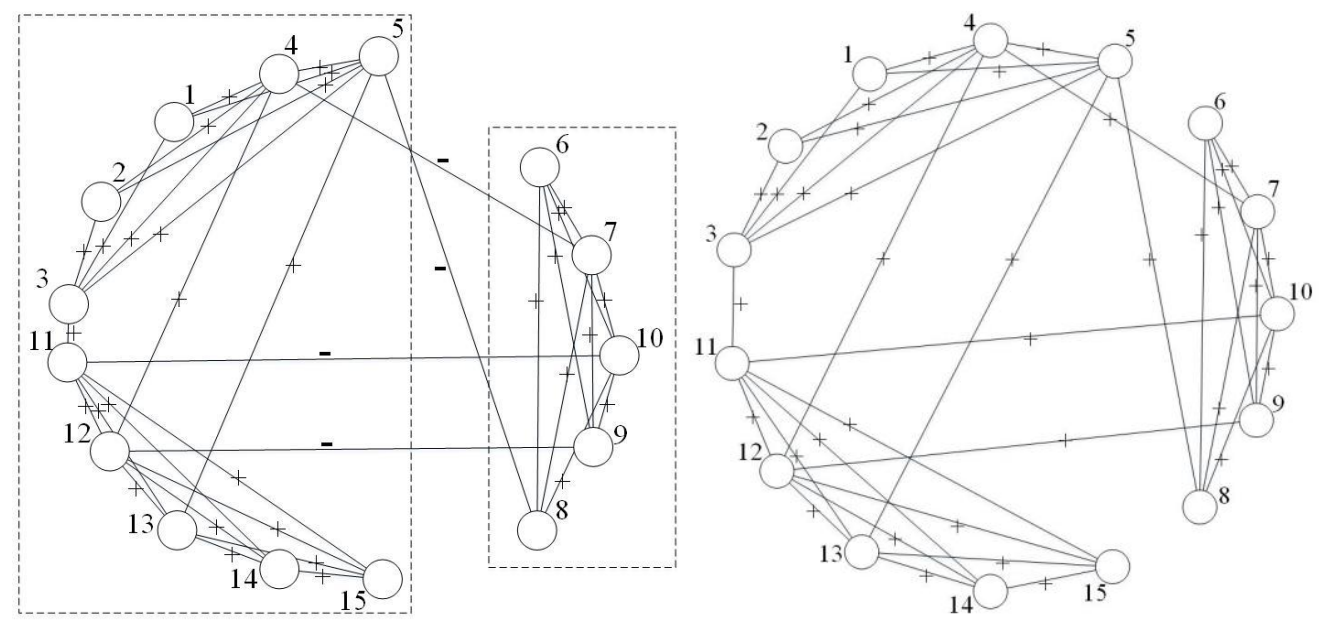

(b) Changing edges into opposite 


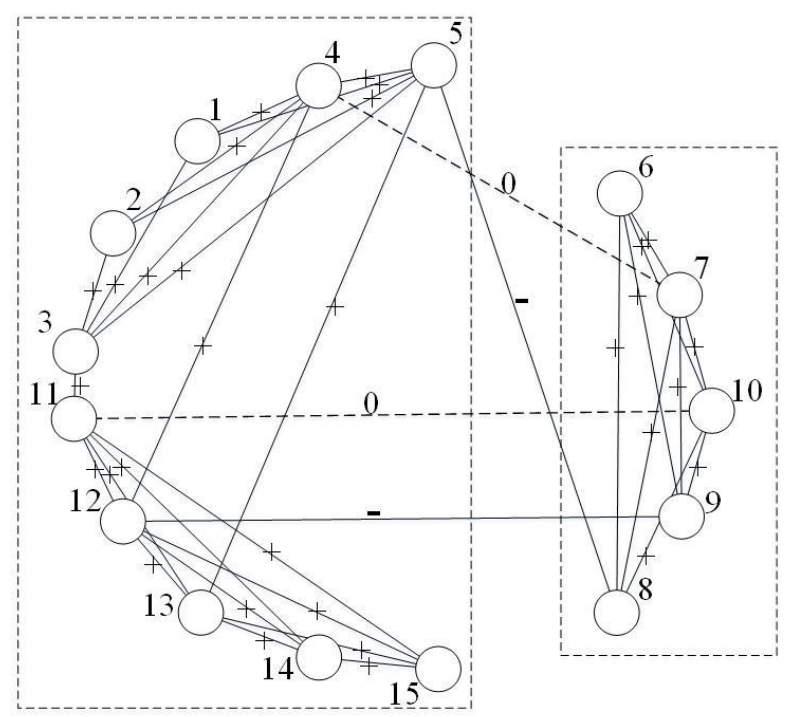

(c) Putting edges into potential

Fig. 5. Transformations of the GN network. The number near the node is the sequence number of the node. Solid lines with '+' denote positive edges; solid lines with '-' denote negative edges; dotted lines with ' 0 ' denote potential edges, and the two dotted frames in (b) and (c) are the two clusters of the balanced network.

Table 2. Mean values of different indexes of 20 runs on GN network with $\mu=1$.

\begin{tabular}{cllllllllll}
\hline Operations & Index & $\omega=0.05$ & $\omega=0.10$ & $\omega=0.15$ & $\omega=0.20$ & $\omega=0.25$ & $\omega=0.30$ & $\omega=0.35$ & $\omega=0.40$ & $\omega=0.45$ \\
\hline & $N_{p n}$ & 2.90 & 1 & 2.60 & 1.80 & 1.05 & 0 & 0 & 0 & 0 \\
including & $N_{n p}$ & 1.05 & 1.45 & 0.70 & 0.95 & 0.35 & 0 & 0 & 0 & 0 \\
Oedges & $N_{p 0}$ & 0 & 0.05 & 0.15 & 0.15 & 0.65 & 2 & 1.25 & 2.20 & 1.75 \\
& $N_{n 0}$ & 0 & 0 & 0 & 0 & 0.95 & 1.30 & 1.4 & 1 & 1.30 \\
& $F$ & 0.20 & 0.27 & 0.55 & 0.60 & 0.75 & 0.66 & 0.40 & 0.32 & 0.15 \\
& $N_{p n}$ & 3.60 & 3.60 & 2.80 & 2.05 & 0.95 & 1 & 0.80 & 0.90 & 0.80 \\
including & $N_{n p}$ & 1.05 & 1.40 & 1 & 0.65 & 1.45 & 1.45 & 1.20 & 1.10 & 1.20 \\
Oedges & $N_{p 0}$ & 0 & 0 & 0 & 0 & 0 & 0 & 0 & 0 & 0 \\
& $N_{n 0}$ & 0 & 0 & 0 & 0 & 0 & 0 & 0 & 0 & 0 \\
& $F$ & 0.23 & 0.50 & 0.57 & 0.54 & 0.60 & 0.74 & 0.70 & 0.80 & 0.90 \\
\hline
\end{tabular}

4.2. Experimental results for four real-world networks

We tested our method on four networks: Slovene parliamentary party network (SPP), illustrative signed network (ISN), epidermal growth factor receptor pathway network (EGN), and macrophage network (MN). For SPP, the network consists of ten Slovene Parliamentary parties [21]. SKD, ZLSD, SDSS, LDS, ZS-ESS, ZS, DS, SLS, SPS-SNS, and SNS are these parties' abbreviated names. This network contains ten 
nodes and 45 edges. For ISN, the network originally depicted mining communities and consists of 28 nodes and 42 edges [22]. For EGN, the network is the pathway map of epidermal growth factor receptor [23], which contains 330 nodes and 852 edges. The network MN is the molecular interaction map of a macrophage [24]. This network contains 697 nodes and 1,425 edges. In our experiments, we make these four networks symmetric by removing symmetric-incompatible edges, as in [25]. Table 3 summarizes the information about these four networks, where " $n$ ", " $m$ ", " $m^{+}$", " $m$ "” represent the number of nodes, edges, +1 edges and -1 edges respectively.

Table 3. Information about the four networks.

\begin{tabular}{lllll}
\hline Network & $n$ & $m$ & $m^{+}$ & $m^{-}$ \\
\hline SPP & 10 & 45 & 18 & 27 \\
ISN & 28 & 42 & 30 & 12 \\
EGN & 330 & 779 & 515 & 264 \\
MN & 697 & 1425 & 947 & 478 \\
\hline
\end{tabular}

In the experiments, we chose $\mu=0.1,1,10,100$, and $\omega=0.05,0.1,0.15,0.2,0.25$, $0.3,0.35,0.4,0.45$, and ran our proposed algorithm on those four networks 20 times with each of the different values of $\mu$ and $\omega$.

Table 4 shows the results for different $\mu$ values. $E_{\text {mean }}$ and $E_{\text {min }}$ represent the mean and the minimum values of energy function of 20 runs on the four networks. From Table 4, we see that it is difficult for $E(s)$ to reach 0 when $\mu=0.1$, especially for EGN and MN. In this situation, the network cannot be transformed into a balanced one because the cost of constraint is too small. For EGN and MN, when $\mu$ gets bigger, $E(s)$ again cannot reach 0 . Since the cost of the energy function is too great, $E(s)$ converges too quickly and the model falls into an unbalanced local minimum. Therefore, $\mu$ should be neither too small nor too big. 
Table 4. Mean values and minimum values of the energy function over 20 runs on the four networks with $\omega=0.15,0.25,0.35$ and different $\mu$ values.

\begin{tabular}{|c|c|c|c|c|c|c|c|c|c|c|c|c|c|}
\hline \multirow{2}{*}{ Networks } & \multirow{2}{*}{ Index } & \multicolumn{3}{|c|}{$\mu=0.1$} & \multicolumn{3}{|c|}{$\mu=1$} & \multicolumn{3}{|c|}{$\mu=10$} & \multicolumn{3}{|c|}{$\mu=100$} \\
\hline & & $\omega=0.15$ & $\omega=0.25$ & $\omega=0.35$ & $\omega=0.15$ & $\omega=0.25$ & $\omega=0.35$ & $\omega=0.15$ & $\omega=0.25$ & $\omega=0.35$ & $\omega=0.15$ & $\omega=0.25$ & $\omega=0.35$ \\
\hline \multirow{2}{*}{ SPP } & $E_{\text {mean }}$ & 2 & 2 & 2 & 0 & 0 & 0 & 0 & 0 & 0 & 0 & 0 & 0 \\
\hline & $E_{\min }$ & 2 & 2 & 2 & 0 & 0 & 0 & 0 & 0 & 0 & 0 & 0 & 0 \\
\hline \multirow{2}{*}{ ISN } & $E_{\text {mean }}$ & 0 & 0.55 & 0.95 & 0 & 0.05 & 0 & 0 & 0 & 0 & 0 & 0 & 0 \\
\hline & $E_{\min }$ & 0 & 0 & 0 & 0 & 0 & 0 & 0 & 0 & 0 & 0 & 0 & 0 \\
\hline \multirow{2}{*}{ EGN } & $E_{\text {mean }}$ & 156 & 162 & 104 & 0.05 & 3.95 & 3.25 & 15 & 10.2 & 3.15 & 18.2 & 9 & 4.15 \\
\hline & $E_{\min }$ & 42 & 123 & 91 & 0 & 0 & 0 & 5 & 3 & 0 & 9 & 2 & 0 \\
\hline \multirow{2}{*}{$\mathrm{MN}$} & $E_{\text {mean }}$ & 146 & 204 & 177 & 0.1 & 14.8 & 12.8 & 37.8 & 22.4 & 9.8 & 37.4 & 26 & 10.4 \\
\hline & $E_{\min }$ & 13 & 149 & 133 & 0 & 3 & 6 & 11 & 11 & 3 & 18 & 13 & 4 \\
\hline
\end{tabular}


In Table 5, we set $\mu=1$ to test the results for different values of $\omega . N_{p n}$ and $N_{n p}$ decrease as $\omega$ increases, while $N_{p 0}$ and $N_{n 0}$ increase as $\omega$ increases. The mean value of the objective function does not increase monotonically as the cost of changing signs of edges increase. The results indicate that our algorithm has chosen the lower-cost solutions.

Table 5. Mean values of different indexes for 20 runs on the four networks with $\mu=1$. Results for different $\omega$ values.

\begin{tabular}{|c|c|c|c|c|c|c|c|c|c|c|}
\hline Networks & Index & $\omega=0.05$ & $\omega=0.10$ & $\omega=0.15$ & $\omega=0.20$ & $\omega=0.25$ & $\omega=0.30$ & $\omega=0.35$ & $\omega=0.40$ & $\omega=0.45$ \\
\hline \multirow[t]{5}{*}{ SPP } & $N_{p n}$ & 2.6 & 1.3 & 1.15 & 0.85 & 0.1 & 0 & 0 & 0 & 0 \\
\hline & $N_{n p}$ & 9.05 & 6.55 & 5.65 & 1.9 & 1.35 & 0.1 & 0 & 0 & 0 \\
\hline & $N_{p 0}$ & 0 & 0 & 0 & 0 & 0 & 0 & 0 & 0 & 0 \\
\hline & $N_{n 0}$ & 0.35 & 0.65 & 1.9 & 2.45 & 5.9 & 5.3 & 5.4 & 7 & 7.7 \\
\hline & $F$ & 0.74 & 1.05 & 1.69 & 1.32 & 2.06 & 1.21 & 0.86 & 0.76 & 0.39 \\
\hline \multirow[t]{5}{*}{ ISN } & $N_{p n}$ & 5.85 & 6.65 & 5.10 & 5.10 & 2.75 & 0.60 & 0 & 0 & 0 \\
\hline & $N_{n p}$ & 5.45 & 4.60 & 3.20 & 2.65 & 0.80 & 0 & 0 & 0 & 0 \\
\hline & $N_{p 0}$ & 0 & 0.05 & 0.05 & 0.15 & 2.45 & 3.10 & 3.80 & 3.10 & 4 \\
\hline & $N_{n 0}$ & 0 & 0.10 & 0.95 & 1.05 & 4.45 & 3.70 & 4.75 & 3.80 & 5.05 \\
\hline & $F$ & 0.565 & 1.19 & 1.60 & 1.91 & 2.66 & 1.54 & 1.28 & 0.69 & 0.45 \\
\hline \multirow[t]{5}{*}{ EGN } & $N_{p n}$ & 202.65 & 194.6 & 202.55 & 190 & 188.15 & 137.20 & 97.50 & 63.55 & 22.80 \\
\hline & $N_{n p}$ & 140.60 & 139.65 & 140.30 & 103.55 & 67.30 & 41.45 & 32.45 & 18.50 & 7.30 \\
\hline & $N_{p 0}$ & 0 & 0 & 0.05 & 3.50 & 25.05 & 101.85 & 197.25 & 306.70 & 412.70 \\
\hline & $N_{n 0}$ & 0.050 & 0.15 & 6.30 & 65.10 & 115.65 & 156.85 & 170.90 & 211.50 & 238.55 \\
\hline & $F$ & 17.19 & 33.49 & 53.70 & 82.24 & 102.98 & 110.69 & 103.96 & 87.39 & 47.51 \\
\hline \multirow[t]{5}{*}{$\mathrm{MN}$} & $N_{p n}$ & 386.7 & 394.25 & 391.80 & 384.10 & 369.50 & 291.15 & 213.55 & 162.95 & 85.55 \\
\hline & $N_{n p}$ & 260.05 & 263.40 & 257.90 & 222.05 & 145.85 & 96.25 & 79.40 & 49.50 & 20.15 \\
\hline & $N_{p 0}$ & 0 & 0 & 0 & 3.20 & 42.7 & 175.3 & 357.35 & 519.2 & 699.3 \\
\hline & $N_{n 0}$ & 0 & 0.10 & 2.65 & 63.95 & 182.3 & 259.55 & 293.75 & 361.85 & 420.90 \\
\hline & $F$ & 32.34 & 65.81 & 98.48 & 145.23 & 199.84 & 220.94 & 212.95 & 183.24 & 108.38 \\
\hline
\end{tabular}

By decoding the chromosomes produced by our algorithms, we finally acquire the optimal solutions of these four real-world networks. For the SPP network, we find two edges leading to global imbalance. One is the negative edge between ZS-ESS and SNS, while the other is the negative edge between DS and SNS. When $\omega<0.25$, we find that these two negative edges tend to change to positive ones. When $\omega=0.25$, there exist four 
best solutions which are: both of the two edges change to positive; both of the two edges change to potential; one of the two edges changes to positive, while the other one changes to potential. When $\omega>0.25$, the two negative edges tend to change to potential ones. For ISN network, the minimum value of the objective function is 0 , which means the original ISN network is balanced, and different changing procedures do not differ in performance; the best solutions for this network are same, because edges in this network do not need to be changed. For EGN and MN networks, we can also find reasonable solutions to achieve the global balance. However, it is difficult for these two networks to achieve the best solutions because of the high computing complexity of the proposed algorithm.

\section{Conclusions}

In this paper, we optimize the transformation of unbalanced networks with a memetic algorithm. Stimulated by Easley and Kleinberg's "filling-in" edges, we optimize the structural balance by not only changing the signs of unbalanced relationships but also changing unbalanced into potential relationships. Applying both procedures gives more solutions and costs less. In order to formalize the transformation of unbalanced networks, a new energy function is employed in our mathematical model, which we combine with a memetic algorithm. The experimental results show that our algorithm can optimize transformations of unbalanced networks efficiently, and with different parameters or procedures, the results may be different. We have not considered here any node attributes that may affect the transformation process, for example, people who have the same habits or point of views may be more likely to 
become friends. In addition, the structural balance has two forms: a strong form (a balanced network can be divided into two clusters) and a weak form (a balanced network can be divided into more than two clusters) [4]. The structural balance we discussed in this paper is the strong form, and the weak form might be explored in the future.

\section{References}

[1] P. Doreian, A. Mrvar, Partitioning signed social networks, Social Networks. 31 (1) (2009) 1-11.

[2] F. Heider, Attitudes and cognitive organization, J. Psychol. 21 (1) (1946) 107-112.

[3] D. Cartwright, F. Harary, Structural balance: a generalization of Heider's theory, Psychol. Rev. 63 (5) (1956) 277-292.

[4] D. Easley, J. M. Kleinberg, Networks, Crowds, and Markets: Reasoning about a Highly Connected World, Cambridge University Press, 2010

[5] R. Nishi, N. Masuda, Dynamics of social balance under temporal interaction, EPL. 107 (4) (2014) 1-6

[6] T. Antal, P. L. Krapivsky, S. Redner, Dynamics of social balance on networks. Phys Rev E. 72 (3) (2005) 036121.

[7] K. Kutakowski, P. Gawronski, P. Gronek, The Heider balance - a continuous approach. Int J Mod Phys C. 16 (5) (2005) 707-716.

[8] S. A. Marvel, S. H. Strogatz, J. M. Kleinberg, Energy landscape of social balance. Phys Rev Lett. 103 (19) (2009) 198701.

[9] V. A. Traag, P. Van Dooren, and P. De Leenheer, Dynamical models explaining social balance and evolution of cooperation, PLoS ONE. 8 (4) (2013) e60063. 
[10] T. H. Summers, I. Shames, Active influence in dynamical models of structural balance in social networks, EPL (Europhysics Letters). 103 (1) (2013) 18001.

[11] G. Facchetti, G. Iacono, C. Altafini, Computing global structural balance in large-scale signed social networks, Proc. Natl. Acad. Sci. 108 (52) (2011) 20953-20958.

[12] R. Figueiredo, Y. Frota, The maximum balanced subgraph of a signed graph: Applications and solution approaches, EUROPEAN JOURNAL OF OPERATIONAL RESEARCH. 236 (2014) 473-487

[13] M. Brusco, D. Steinley, A Tabu-Search Heuristic for Deterministic Two-Mode Blockmodeling of Binary Network Matrices, PSYCHOMETRIKA. 76 (4) (2011) 612-633

[14] B. J. Ross, E. Zuviria, Evolving dynamic Bayesian networks with multi-objective genetic algorithms, APPLIED INTELLIGENCE. 26 (1) (2007) 13-23

[15] F. Barahona, On the computational complexity of Ising spin glass models, J. Phys. A: Math. Gen. 15 (10) (1982) 3241.

[16] D. W. Gong, N. N. Qin, X. Y. Sun, Evolutionary algorithms for optimization problems with uncertainties and hybrid indices, Information Sciences, 181 (19) (2011) 4124-4138.

[17] J. F. Xuan, H. Jiang, Z. L. Ren, Z. X. Luo, Solving the Large Scale Next Release Problem with a Backbone Based Multilevel Algorithm, IEEE Transactions on Software Engineering, 38 (5) (2012) 1195-1212.

[18] R. Berretta, C. Cotta, P. Moscato, Enhancing the performance of memetic algorithms by using a matching-based recombination algorithm, Metaheuristics: Computer Decision-making, Kluwer Academic Publishers, (2004) 65-90.

[19] L. Ma, M. Gong, J. Liu, Q. Cai, L. Jiao, Multi-level learning based memetic algorithm for 
community detection, Appl. Soft Comput. 19 (2014) 121-133.

[20] Y. Sun, H. Du, M. Gong, Fast computing global structural balance in signed networks based on memetic algorithm, Physica A. 415 (2014) 261-272

[21] A. Ferligoj, A. Kramberger, An analysis of the Slovene parliamentary parties network, Developments in Statistics and Methodology. (1996) 105-144.

[22] B. Yang, W. K. Cheung, J. Liu, Community mining from signed social networks, IEEE Transactions on Knowledge and Data Engineering. 19 (10) (2007) 1333-1348.

[23] K. Oda, Y. Matsuoka, A. Funahashi, H. Kitano, A comprehensive pathway map of epidermal growth factor receptor signaling," Molecular systems biology. 1 (1) (2005).

[24] K. Oda, T. Kimura, Y. Matsuoka, A. Funahashi, M. Muramatsu, H. Kitano, Molecular interaction map of a macrophage, AfCS Research Reports. 2 (14) (2004) 1-12.

[25] G. Iacono, F. Ramezani, N. Soranzo, C. Altani, Determining the distance to monotonicity of a biological network: a graph-theoretical approach, IET Systems Biology. 4 (3) (2010) 223-235. 